\title{
Development of a Simulation Tool for "Real World” SEM Applications
}

\section{Drouin and A.R. Couture}

${ }^{1}$ Départmenet de génie électrique et génie informatique, Université de Sherbrooke, Sherbrooke, Québec, J1K 2R1, Canada.

The topic of this paper is to present a powerful tool for SEM analysis. The Monte Carlo program CASINO (www.gel.usherb.ca/casino) as been adapted to be able to reproduce more adequately real sample used in SEM. Simple geometry such as vertical and horizontal planes used in the previous version limits considerably the range of applications of this tool. The goal of this version is to be able to simulate backscattered electron image and profiles of a complex geometry such as one shown in Figure 1-a. This demonstration sample, is composed of spherical gold particles of various diameter embedded in a silicon matrix at different depth. Rough surface, such a one shown in figure 1-b, can also be created with a 3-D design software and simulated in CASINO.

The program is using complex algorithms to track down the electron position as it travels within the sample. A tree like algorithm is first used to subdivide the samples composed of a large number of triangles in cubes containing a few of them. This technique reduces considerably the computing time needed to locate the position of an electron collision in the sample. Each triangle composing the sample defines a boundary between different regions of different chemical composition. As the electron travels throw those regions the electron mean free path is corrected.

The core of the electron trajectory computation is based on a single scattering model. Tabulated Mott elastic cross-sections are used due to fast calculation and accurate interaction at low beam energy. A continuous energy loss model is implemented based on the modify version of Bethe law by Joy and Luo.

Figure 2 shows an example of backscattered image computed using CASINO by scanning the beam across the sample of figure 1-a. A matrix of 50x50 points were used to create this image. The simulation conditions are as follow; $5 \mathrm{keV}$ electron energy, $10 \mathrm{~nm}$ beam diameter, 2000 simulated electrons per points. Figure 3 shows a backscattered image of the same sample but simulated at 10 $\mathrm{keV}$, this image reveal deeper embedded gold sphere. Figure 4, shows another example of simulated backscattered electron images of the rough surface shown in figure 1-b. The sample is bulk InGaAs and simulation conditions were $1 \mathrm{keV}$ electron energy, $10 \mathrm{~nm}$ beam diameter, 2000 electrons per point. By increasing the number of simulated electron per point to 10000 , the image noise is reduced considerably, figure 5. For this sample, 36192 triangles were used to represent the sample and 2500 simulated point were performed to create the image. The total simulation time is approximately 3 hours with a AMD K7@ $600 \mathrm{MHz}$ computer.

\section{References}

D. Drouin, A.R. Couture, R. Gauvin, Microscopy and Microanalysis, Vol.7, Sup.2, 684-685.

D. Drouin, A.R. Couture, R. Gauvin, P. Horny, Scanning, Vol. 23, 2, 93-94, (2001).

D. Drouin, P. Hovington et R. Gauvin, Scanning, 19, 20-28, (1997)

D. Joy and S. Luo, Scanning, $\underline{11}, 176-180,(1989)$ 


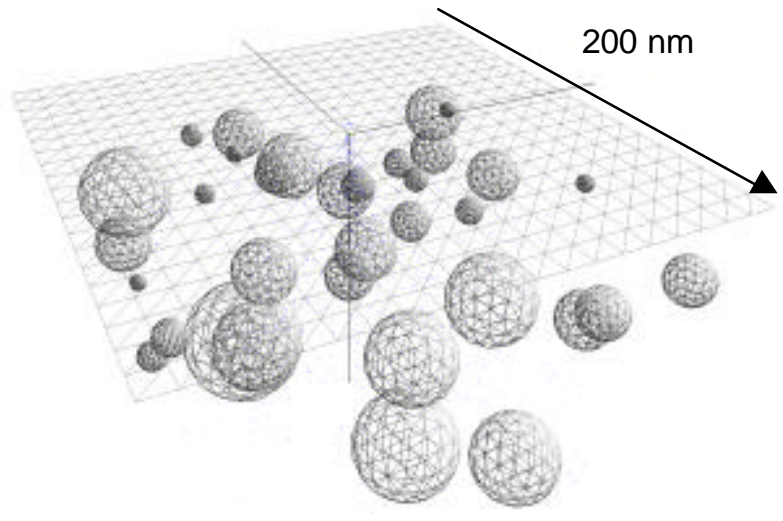

Figure 1-a Demonstration sample composed of gold particles embedded in silicon matrix.

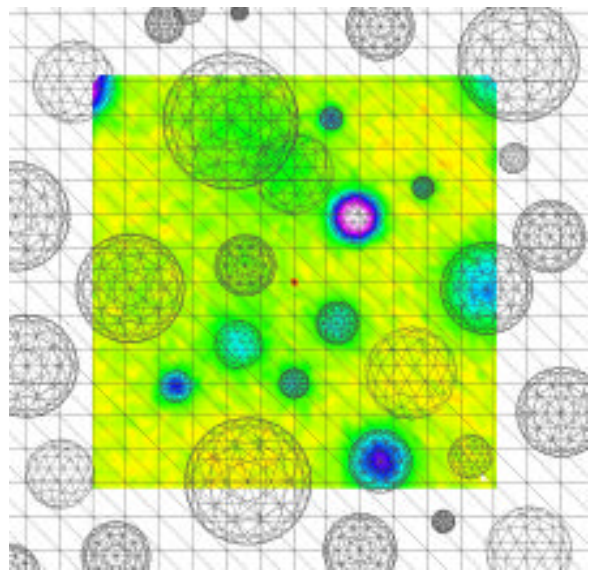

Figure 2 Simulated backscattered image (50 points $\mathrm{x} 50$ points) of figure 1-a sample. $\mathrm{E}_{0}=5 \mathrm{keV}$, number of electrons 2000. Total width of color image $150 \mathrm{~nm}$
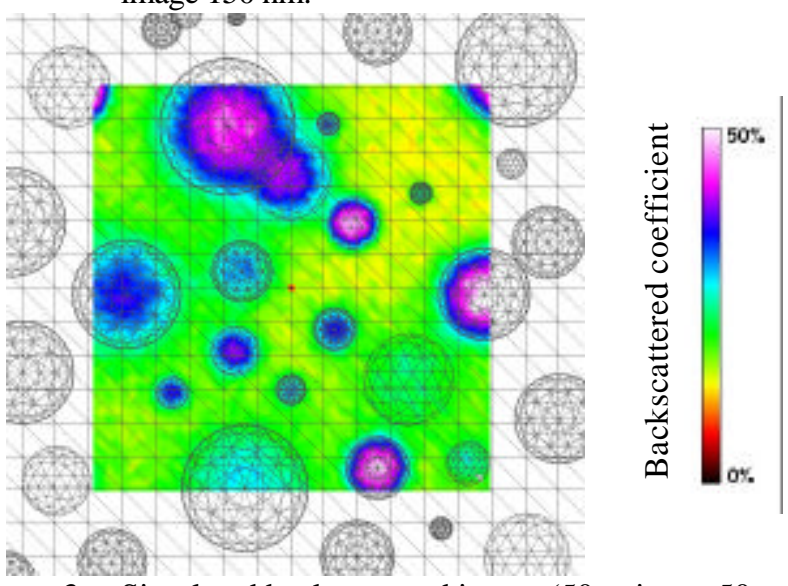

Figure 3 Simulated backscattered image (50 points x 50 points) of figure 1-a sample. $\mathrm{E}_{0}=10 \mathrm{keV}$, number of electrons 2000 .

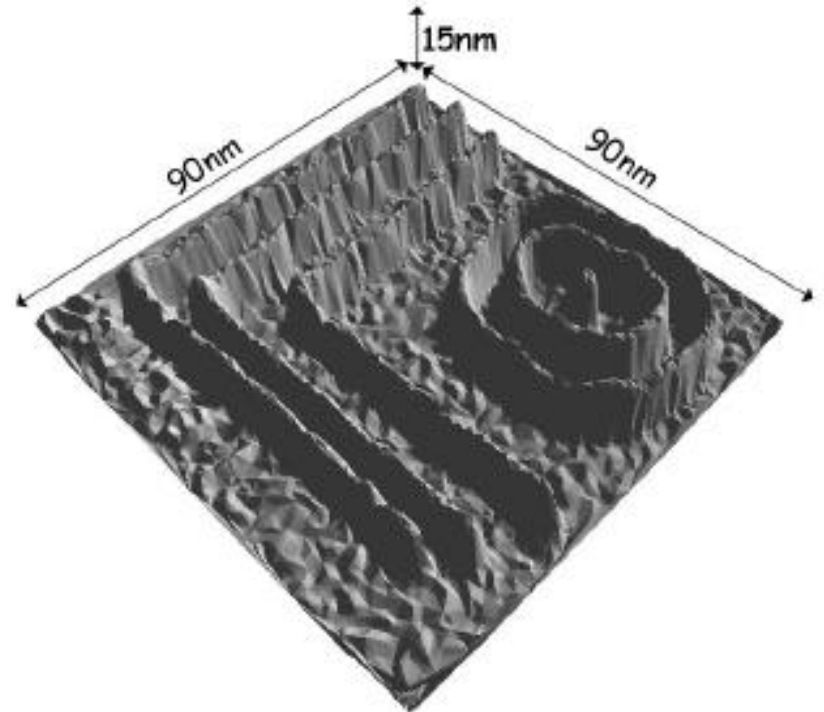

Figure 1-b Rough surface of InGaAs composed of 36192 triangles.
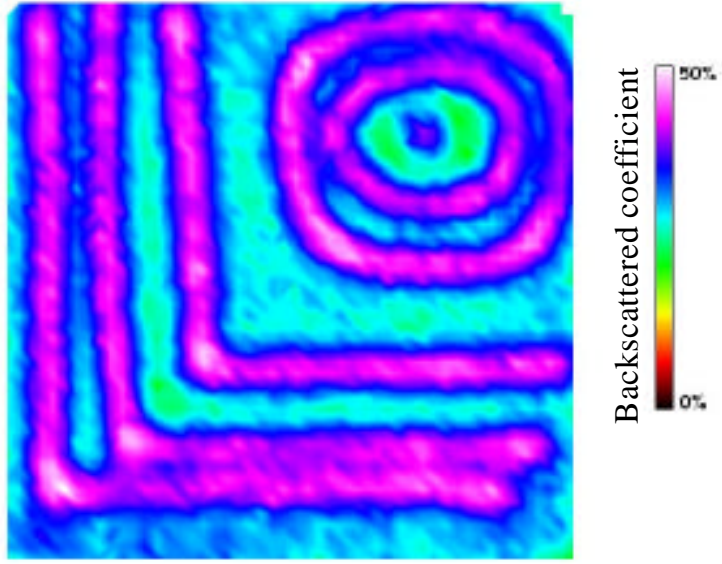

Figure 4 Simulated backscattered image ( 50 points $x 50$ points) of figure 1-b sample. $\mathrm{E}_{0}=1 \mathrm{keV}$, number of electrons 2000 .
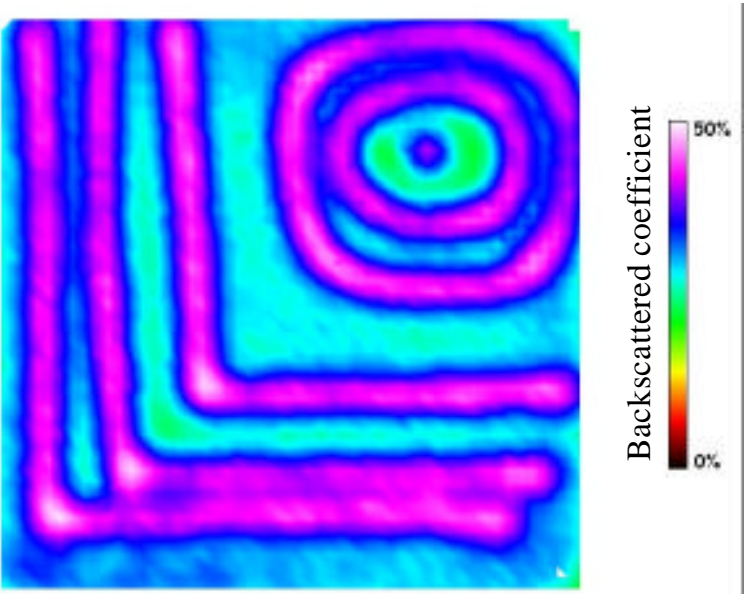

Figure 5 Simulated backscattered image ( 50 points $x 50$ points) of figure 1-b sample. $\mathrm{E}_{0}=1 \mathrm{keV}$, number of electrons 10000. 